\title{
Design of a Three Degrees of Freedom Robotic Arm
}

\author{
Madiha Farman \\ Department of Mechanical \\ Engineering \\ Khalifa University of \\ Science \& Technology \\ Abu Dhabi, UAE
}

\author{
Muneera Al-Shaibah \\ Department of Mechanical \\ Engineering \\ Khalifa University of \\ Science \& Technology \\ Abu Dhabi, UAE
}

\author{
Zoha Aoraiath \\ Department of Mechanical \\ Engineering \\ Khalifa University of \\ Science \& Technology \\ Abu Dhabi, UAE
}

\author{
Firas Jarrar \\ Department of Mechanical \\ Engineering \\ Khalifa University of \\ Science \& Technology \\ Abu Dhabi, UAE
}

\begin{abstract}
This paper concerns with the design of a three degrees of freedom robotic arm, which is intended to pick and place lightweight objects based on a color sorting mechanism. It is mainly made of three joints, a gripper, two rectangular shaped links, a rotary table and a rectangular platform. The angular rotation of each joint is powered by a servomotor. Furthermore, the angular position of each servomotor shaft is controlled by a signal from an Arduino microcontroller which executes a Matlab code. The Matlab code includes the inverse kinematics equations which are necessary for the determination of the target joint angles for a certain Cartesian position of the end-effector. The robotic arm's design process included several static and dynamic calculations, mechanical properties calculations and prototype testing in order to provide a final product with well-established structure and functionalities.
\end{abstract}

\section{General Terms}

Robotics

\section{Keywords}

Robotic arm, three degrees of freedom, forward kinematics, inverse kinematics, workspace

\section{INTRODUCTION}

Using robots over human labor to perform accurate and precise work is becoming more preferable, due to its better performance and minimal risks. An articulated robotic arm consists of links that connect a certain number of rotary joints in series to an end effector. The number of joints represents the number of Degrees of Freedom (DOF) of the robotic arm. The joints are usually actuated using servomotors, which provide the necessary torque to rotate the attached links. Microcontrollers are used to send the electrical signals required for controlling the angular motion of the servomotor shafts.

In order to construct a robotic arm capable of performing the intended tasks accurately and efficiently, proper design and simulation are deemed necessary. In this paper, the design procedure and calculations are presented for the three DOF robotic arm shown in Figure 1. An example of the use of a robotic arm similar to the one considered in this paper is to color sort objects such as test tubes in medical biology laboratories. The forward and inverse kinematics of the three DOF robotic arm was established. The former describes the position and orientation of the end effector for given rotation angles. The latter, the inverse kinematics, determines the joint angles required to reach the desired position and orientation of the end effector. Furthermore, the static torques, the most appropriate link cross sections, and the workspace were determined.
The study of robotic arms involves nonlinear analysis and includes challenges related to the existence of multiple solutions, singularity points and possible redundancies. There are several studies found in open literature that consider the kinematic analysis of robotic arms. For example, Shah et al. [1] carried out a forward kinematic analysis of a five DOF Pravak robotic arm. Their procedure included calculating the Denavit-Hartenberg (D-H) parameters [2] and the homogenous transform matrices. However, they did not consider the inverse kinematics analysis. In another study, Fernini [3] considered a two DOF articulated robotic arm. He used both SolidWorks and Matlab to check the theory and the robot motion simulation. Fernini considered both the forward and the inverse kinematics and the expended energy during motion. Subhashin et al. [4] investigated a SCARA robotic arm. They derived the forward and inverse kinematic equations and simulated the robotic arm using CAD software and Matlab.

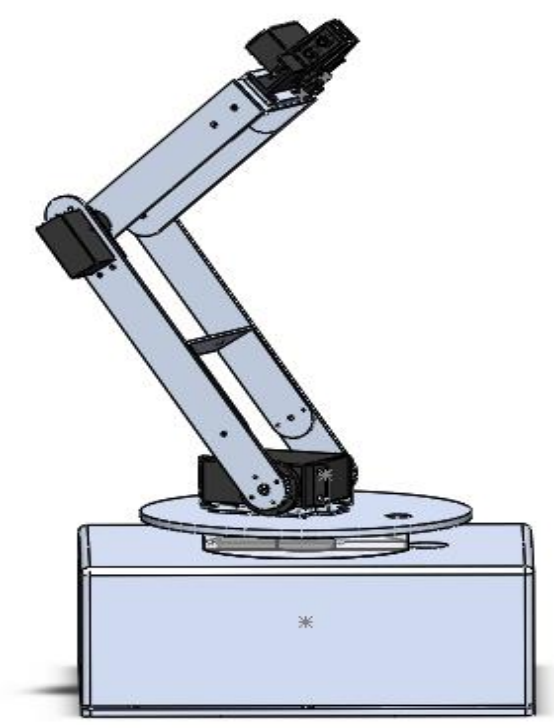

Figure 1: A SolidWorks model of the considered 3 DOF robotic arm

\section{DESIGN CALCULATIONS}

\subsection{Objectives}

First of all, well-defined objectives were identified as shown in the objective tree in Figure 2. The conceptual design process included several stages that led to the final design presented in Figure 1. This design of the robotic arm consists of two links, three joints, a parallel plate gripper, a rotary table, an acrylic circular disc and a rectangular platform. The links are pairs of two rectangular beams structures connected by brackets. There are two U-shaped brackets used for 
connecting parts. The rectangular platform is intended to stabilize the rotary table and support the whole structure. The acrylic disc is used to mount the rotary table to the platform. All of the structural parts are made of Aluminum.

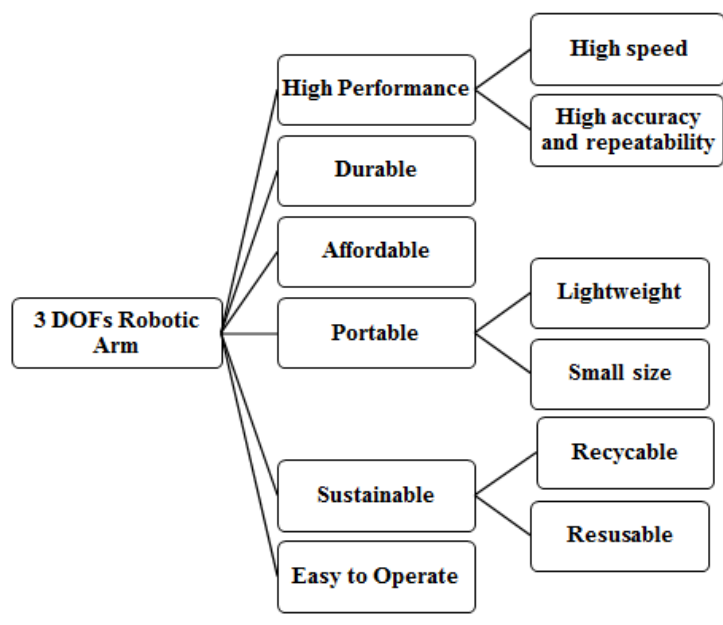

Figure 2: The design objectives tree

The robotic arm consists of three joints; the waist joint represented by rotation of the rotary table, the shoulder joint represented by the rotation of Link (1) and the elbow joint represented by Link (2)'s rotation. Each joint employs a specific servomotor to provide the torque required for the motion. Each motor receives the shaft position controlling signal from a Matlab code, through an Arduino microcontroller. The Cartesian position of the object to be picked up, which is identified by its color, is detected by using a mobile camera and an available Matlab plug-in. The inverse kinematics equations are then used to find the required joint angles corresponding to the aforementioned coordinates. Before constructing the robotic arm prototype, it was necessary to carry out certain calculations such as to determine the required torque, the forward and inverse kinematics, and the robotic arm workspace.

\subsection{Section Modulus}

In order to determine the most appropriate cross sectional shape of the robotic arm's links, three possible geometries were considered; a solid cylindrical beam, a hollow cylindrical beam and a set of two rectangular beams. The section modulus, $S$, is a geometrical property, which indicates the strength of beams based on their cross sectional shapes or areas.

$S=\frac{I}{c}$

where $I$ is the second moment of area $\left(\mathrm{mm}^{4}\right)$, and $c$ is the maximum distance from the neutral axis $(\mathrm{mm})$.

It can be seen from the generated graph, shown in Figure 3, that for any value of the cross sectional area, the set of two parallel rectangular beams has the highest value of $S$, followed by the hollow cylindrical beam and then lastly, the solid cylindrical beam. This means that the set of two parallel rectangular beams have the highest structural strength to weight ratio out of the three links, considering they are all made of the same material. Hence, rectangular beams were used for the robotic arm's links.

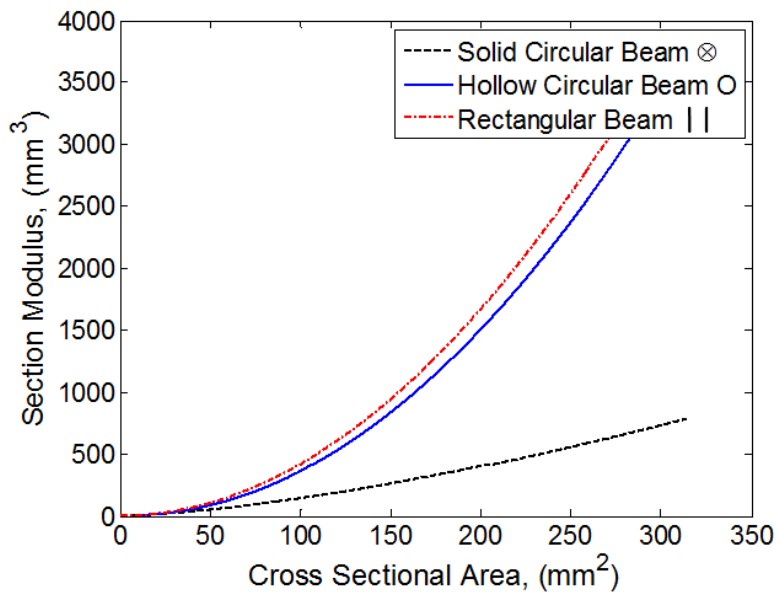

Figure 3: The section modulus against cross sectional area for the three considered beams

\subsection{Static Torque}

The following calculation was carried out in order to ensure that the torque of the elbow and shoulder motors will be enough to rotate the joints with a maximum payload of $200 \mathrm{~g}$. The maximum required torque corresponding to the worst case scenario with the arm fully stretched out is shown in Figure 4. From the moments equilibrium equations, Eqs. 2 and 3 , the results showed that a minimum of $0.351 \mathrm{~N}$.m for the elbow and $1.259 \mathrm{~N} . \mathrm{m}$ for the shoulder are required. Consequently, based on the available servomotors in the market, the selected servomotors for the elbow and shoulder joints provide a maximum torque of $0.941 \mathrm{~N} . \mathrm{m}$ and $2.422 \mathrm{~N} . \mathrm{m}$, respectively.

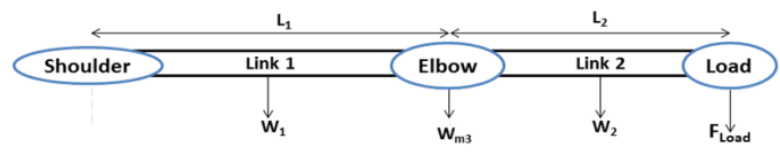

Figure 4: Free body diagram of the robotic arm in the fully stretched out configuration

$T_{\text {elbow }}=\left[F_{\text {load }} \times L_{2}\right]+\left[w_{2} \times\left(\frac{L_{2}}{2}\right)\right]$

(2)

$T_{\text {shoulder }}=\left[F_{\text {load }} \times\left(L_{1}+L_{2}\right)\right]+\left[W_{2} \times\left(\frac{L_{2}}{2}+L_{1}\right)\right]+$

$\left(W_{m 3} \times L_{1}\right)+\left(W_{1} \times \frac{L_{1}}{2}\right)$

Where $W_{1}$ and $W_{2}$ represent the weight of the Links (1) and (2), respectively. $W_{m 3}$ accounts for the weight of elbow motor. $F_{\text {load }}$ represents the combined weight of the payload and the gripper.

\subsection{Forward Kinematics}

Five frames were assigned to the robotic arm as shown in Figure 5. The frame assignment procedure followed can be summarized in three steps [5]:

- Assign the $\mathrm{Z}_{\mathrm{i}}$ axis pointing along the $\mathrm{i}$-th joint axis (either one of two choices). It is better to assign $Z_{0}$ and $Z_{n+1}$ parallel to $Z_{1}$ and $Z_{n}$, respectively.

- Assign the $X_{i}$ axis pointing along the common perpendicular from $Z_{i}$ to $Z_{i+1}$.

- Assign the $Y_{i}$ axis according to the right hand rule.

The non-rotating reference Frame $\{0\}$ was attached to a fixed point at the center of the rotary base. Frame $\{1\}$ 
corresponding to Link (1) was attached at its upper end. Note that the origin of this frame could have been appointed to any location along Link (1) since $\mathrm{Z}_{0}$ and $\mathrm{Z}_{1}$ are parallel to each other. Frame $\{2\}$ rotates with Link (2) and is located at the intersection between Link (1) and Link (2), thus having $X_{2}$ along the common perpendicular pointing from $\mathrm{Z}_{2}$ to $\mathrm{Z}_{3}$. Frame $\{3\}$ rotates with Link (3) and is attached at its intersection with Link (2). Finally, Frame $\{4\}$ corresponding to the end-effector is located midway between the two grippers.

Based on the defined frames, the Denavit-Hartenberg (D-H) parameters [2] were determined as shown in Table 1.

Table 1: The D-H parameters

\begin{tabular}{|c|c|c|c|c|}
\hline $\boldsymbol{i}$ & $\boldsymbol{\alpha}_{\boldsymbol{i}-\boldsymbol{1}}$ & $\boldsymbol{a}_{\boldsymbol{i}-\boldsymbol{1}}$ & $\boldsymbol{d}_{\boldsymbol{i}}$ & $\boldsymbol{\theta}_{\boldsymbol{i}}$ \\
\hline 1 & $0^{o}$ & 0 & $L_{1}$ & $\theta_{1}$ \\
\hline 2 & $90^{o}$ & 0 & 0 & $\theta_{2}$ \\
\hline 3 & $0^{o}$ & $L_{2}$ & 0 & $\theta_{3}$ \\
\hline 4 & $0^{o}$ & $L_{3}$ & 0 & $0^{\circ}$ \\
\hline
\end{tabular}

The definition of each of the D-H parameters is as follows:

$\alpha_{i}$ : Link twist is the angle about $\mathrm{X}_{\mathrm{i}}$ between $\left(\mathrm{Z}_{\mathrm{i}}, \mathrm{Z}_{\mathrm{i}+1}\right)$ $a_{i}$ : Link length is the distance along $\mathrm{Xi}$ between $\left(\mathrm{Z}_{\mathrm{i}}, \mathrm{Z}_{\mathrm{i}+1}\right)$

$d_{i}$ : Link offset is the distance along $\mathrm{Z}_{\mathrm{i}}$ between $\left(\mathrm{X}_{\mathrm{i}-1}, \mathrm{X}_{\mathrm{i}}\right)$

$\theta_{i}$ : Joint angle is the angle about $\mathrm{Z}_{\mathrm{i}}$ between $\left(\mathrm{X}_{\mathrm{i}-1}, \mathrm{X}_{\mathrm{i}}\right)$

The D-H parameters were then used to compute the homogenous transform matrices $\left({ }_{1}^{0} T,{ }_{2}^{1} T,{ }_{3}^{2} T\right.$ and $\left.{ }_{4}^{3} T\right)$ by substituting in the general formula for ${ }_{i}^{i-1} T$.

${ }_{i}^{i-1} T=\left[\begin{array}{cccc}c \theta_{i} & -s \theta_{i} & 0 & \alpha_{i-1} \\ s \theta_{i} c \alpha_{i-1} & c \theta_{i} c \alpha_{i-1} & -s \alpha_{i-1} & -s \alpha_{i-1} d_{i} \\ s \theta_{i} s \alpha_{i-1} & c \theta_{i} s \alpha_{i-1} & c \alpha_{i-1} & c \alpha_{i-1} d_{i} \\ 0 & 0 & 0 & 1\end{array}\right]$

${ }_{1}^{0} T=\left[\begin{array}{cccc}c_{1} & -s_{1} & 0 & 0 \\ s_{1} & c_{1} & 0 & 0 \\ 0 & 0 & 1 & L_{1} \\ 0 & 0 & 0 & 1\end{array}\right]$

${ }_{2}^{1} T=\left[\begin{array}{cccc}c_{2} & -s_{2} & 0 & 0 \\ 0 & 0 & -1 & 0 \\ s_{2} & c_{2} & 0 & 0 \\ 0 & 0 & 0 & 1\end{array}\right]$

${ }_{3}^{2} T=\left[\begin{array}{cccc}c_{3} & -s_{3} & 0 & L_{2} \\ s_{3} & c_{3} & 0 & 0 \\ 0 & 0 & 1 & 0 \\ 0 & 0 & 0 & 1\end{array}\right]$

${ }_{4}^{3} T=\left[\begin{array}{cccc}1 & 0 & 0 & L_{3} \\ 0 & 1 & 0 & 0 \\ 0 & 0 & 1 & 0 \\ 0 & 0 & 0 & 1\end{array}\right]$

where $c_{i}$ is the cosine of the angle $\theta_{i}$, and $s_{i}$ represents the sine.

By multiplying the homogeneous transformation matrices in the right order, ${ }_{4}^{0} T$ describing the end-effector frame with the reference frame may be obtained.

${ }_{4}^{0} T={ }_{1}^{0} T{ }_{2}^{1} T_{3}^{2} T_{4}^{3} T$
${ }_{4}^{0} T=\left[\begin{array}{cccc}c_{1} c_{23} & -c_{1} s_{23} & s_{1} & c_{1} c_{23} L_{3}+c_{1} c_{2} L_{2} \\ s_{1} c_{23} & -s_{1} s_{23} & -c_{1} & s_{1} c_{23} L_{3}+s_{1} c_{2} L_{2} \\ s_{23} & c_{23} & 0 & s_{23} L_{3}+s_{2} L_{2}+L_{1} \\ 0 & 0 & 0 & 1\end{array}\right]$

The first three entries of the last column in ${ }_{4}^{0} T$ represent the Cartesian coordinates of the position of the origin of the end effector frame $\{4\}$ with respect to the reference frame $\{0\}$.

$x=c_{1} c_{23} L_{3}+c_{1} c_{2} L_{2}$

$y=s_{1} c_{23} L_{3}+s_{1} c_{2} L_{2}$

$z=s_{23} L_{3}+s_{2} L_{2}+L_{1}$

Notice that $c_{23}$ is the cosine of the sum of $\theta_{2}$ and $\theta_{3}$ and $s_{23}$ is the sine of the sum of these two angles.

The procedure for obtaining the analytical expression for the $x, y$ and $z$ coordinates is called forward kinematics analysis. The forward kinematics, Eqs. 11 to 13, produce the position of the end effector as a function of the joint angles $\theta_{1}, \theta_{2}$ and $\theta_{3}$.

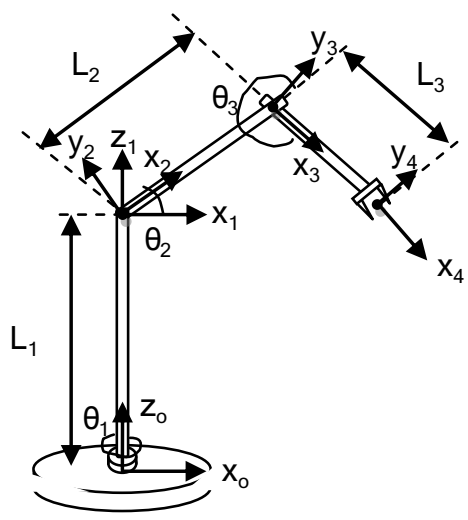

Figure 5: Frame assignments for the kinematics analysis

\subsection{Inverse Kinematics}

In the inverse kinematics, the position coordinates equations, Eqs. 11 - 13, were used to find the analytical expressions of the rotational angles (i.e. $\theta_{1}, \theta_{2}$ and $\theta_{3}$ ) for the waist, shoulder and elbow motors as a function of the desired $x, y$ and $z$ coordinates. The derivation of the inverse kinematic equations is summarized as follows:

Firstly, dividing Eq. 12 by Eq. 11 gives

$\frac{y}{x}=\frac{s_{1} c_{23} L_{3}+s_{1} c_{2} L_{2}}{c_{1} c_{23} L_{3}+c_{1} c_{2} L_{2}}=\frac{s_{1}\left(c_{23} L_{3}+c_{2} L_{2}\right)}{c_{1}\left(c_{23} L_{3}+c_{2} L_{2}\right)}=\frac{s_{1}}{c_{1}}=\tan \theta_{1}$

As a result $\theta_{1}$ is explicitly expressed as

$\theta_{1}=\operatorname{atan} 2\left(\frac{y}{x}\right)$

The (atan2) function generates the arctangent of the argument and the proper quadrant based on the signs of both the denominator and the numerator.

Furthermore, by summing up the squares of Eqs. 11 to 13 and rearranging one can obtain an explicit expression for the cosine of $\theta_{3}$ as a function of the robotic arm geometry and the coordinates of the target point.

$c_{3}=\frac{x^{2}+y^{2}+z^{2}-\left(L_{1}{ }^{2}+L_{2}{ }^{2}+L_{3}{ }^{2}\right)-2 L_{1}\left(z-L_{1}\right)}{2 L_{2} L_{3}}$

From the Pythagorean trigonometric identity

$s_{3}= \pm \sqrt{1-c_{3}}$ 
Notice the ambiguity in algebraic sign in the above equation, Eq. 17, is an indication of multiple solutions. The two solutions correspond to the two different configurations that the arm could take to reach the same point (i.e. elbow up and elbow down). Accordingly from the previous,

$\theta_{3}=\operatorname{atan} 2\left(\frac{s_{3}}{c_{3}}\right)$

Considering Eq. 13, applying the angle addition trigonometric identity, and defining the following variables

$k_{1}=\left(c_{3} L_{3}+L_{2}\right)$

$k_{2}=\left(s_{3} L_{3}\right)$

it can be shown that

$z-L_{1}=k_{1} s_{2}+k_{2} c_{2}$

By rearranging and squaring both sides of the equation,

$\left(z-L_{1}-k_{1} s_{2}\right)^{2}=\left(k_{2} c_{2}\right)^{2}$

Then by expanding the left side and regrouping the terms, the following quadratic equation can be obtained

$\left(k_{1}^{2}+{k_{2}}^{2}\right) s_{2}^{2}+2 k_{1}\left(L_{1}-z\right) s_{2}+\left(z^{2}+L_{1}{ }^{2}-k_{2}{ }^{2}-\right.$

$2 z L 1=0$

Using the quadratic formula, $\theta_{2}$ can be expressed in terms of the robotic arm geometry, the target point $\mathrm{z}$ - coordinate, and the value of $\theta_{2}$ as obtained from Eq. 18

$\theta_{2}=\sin ^{-1}\left(\frac{A \pm \sqrt{B}}{2\left(k_{1}{ }^{2}+k_{2}{ }^{2}\right)}\right)$

where

$A=-2 k_{1}\left(L_{1}-z\right)$

$B=\left(2 k_{1}\left(L_{1}-z\right)\right)^{2}-4\left(k_{1}{ }^{2}+{k_{2}}^{2}\right)\left(z^{2}+L_{1}{ }^{2}-k_{2}{ }^{2}-\right.$

$2 z L 1$

It can be noticed from Eq. 22 that there are two solutions, roots, for $\theta_{2}$. It is noteworthy that there is another approach to find $\theta_{2}$. To obtain this alternative formula for $\theta_{2}$, the $x$ and $y$ coordinates equations, Eqs. 11 and 12, should be rewritten as

$x=c_{1}\left(k_{1} c_{2}-k_{2} s_{2}\right)$

$y=s_{1}\left(k_{1} c_{2}-k_{2} s_{2}\right)$

In obtaining Eqs. 23 and 24, the trigonometric angle addition identity was used. Now by subtracting Eq. 24 from Eq. 23,

$\frac{x-y}{c_{1}-s_{1}}=k_{1} c_{2}-k_{2} s_{2}$

Together with Eq. 19, Eq. 25 constitute a system of two transcendental equations that can be solved by transformation to polar coordinates using

$r=\sqrt{\left(k_{1}\right)^{2}+\left(k_{2}\right)^{2}}$ and $\gamma=\operatorname{atan} 2\left(\frac{k_{2}}{k_{1}}\right)$ to give

$k_{1}=r \cos \gamma \quad$ and $\quad k_{2}=r \sin \gamma$

Thus,

$\frac{x-y}{c_{1}-s_{1}}=r c_{\gamma} c_{2}-r s_{\gamma} s_{2}$

Applying the angle addition trigonometric identity,

$\frac{x-y}{r\left(c_{1}-s_{1}\right)}=\cos \left(\gamma+\theta_{2}\right)$

Notice that Eq. 19, using the same transformation and the angle addition trigonometric identity, can be written as

$\frac{z-L_{1}}{r}=\sin \left(\gamma+\theta_{2}\right)$

Dividing Eq. 28 by Eq. 27 and substituting for the values of $k_{1}, k_{2}, r$ and $\gamma$,

$\theta_{2}=\operatorname{atan} 2\left[\frac{\left(z-L_{1}\right)\left(c_{1}-s_{1}\right)}{(x-y)}\right]-\operatorname{atan} 2\left[\frac{s_{3} L_{3}}{\left(c_{3} L_{3}+L_{2}\right)}\right]$

Equation 29 offers another way to explicitly compute $\theta_{2}$. However, both methods are supposed to produce the same result. Therefore, just by substituting the target $x, y$ and $z$ coordinates into Eqs. 15, 18, and 22 (or 29) will generate $\theta_{1}$, $\theta_{2}$ and $\theta_{3}$, after which the robotic arm links will rotate accordingly to reach that location in space.

\subsection{Workspace}

In this section the workspace is identified analytically by investigating the equations obtained from the forward kinematics calculations. The cosine of an angle lies between a value of -1 and 1 , therefore the following mathematical inequality holds true based on the formula obtained in Eq. 16 ,

$-2 L_{2} L_{3} \leq x^{2}+y^{2}+z^{2}-2 L_{1} z+2 L_{1}{ }^{2}-\left({L_{1}}^{2}+L_{2}{ }^{2}+\right.$ $L 32 \leq 2 L 2 L 3$

With little manipulation of the above equation,

$\left(L_{2}-L_{3}\right)^{2} \leq x^{2}+y^{2}+\left(z-L_{1}\right)^{2} \leq\left(L_{2}+L_{3}\right)^{2}$

The result is an equation of a sphere of a center at $\left(0,0, L_{l}\right)$ with an inner and outer radii of $\left(L_{2}-L_{3}\right)$ and $\left(L_{2}+L_{3}\right)$, respectively. Based on the actual arm's links lengths, the servomotors' angle restrictions and the existence of a base, a $3 \mathrm{D}$ plot that reflects the arm's workspace was generated in Matlab and is shown in Figure 6. In this figure, the workspace is the area enclosed by the blue and red highlighted boundaries. Furthermore, from Figure, it can be shown that the designed arm's minimum and maximum horizontal reach is $\pm 270.8 \mathrm{~mm}$ and $\pm 399.5 \mathrm{~mm}$, respectively. 


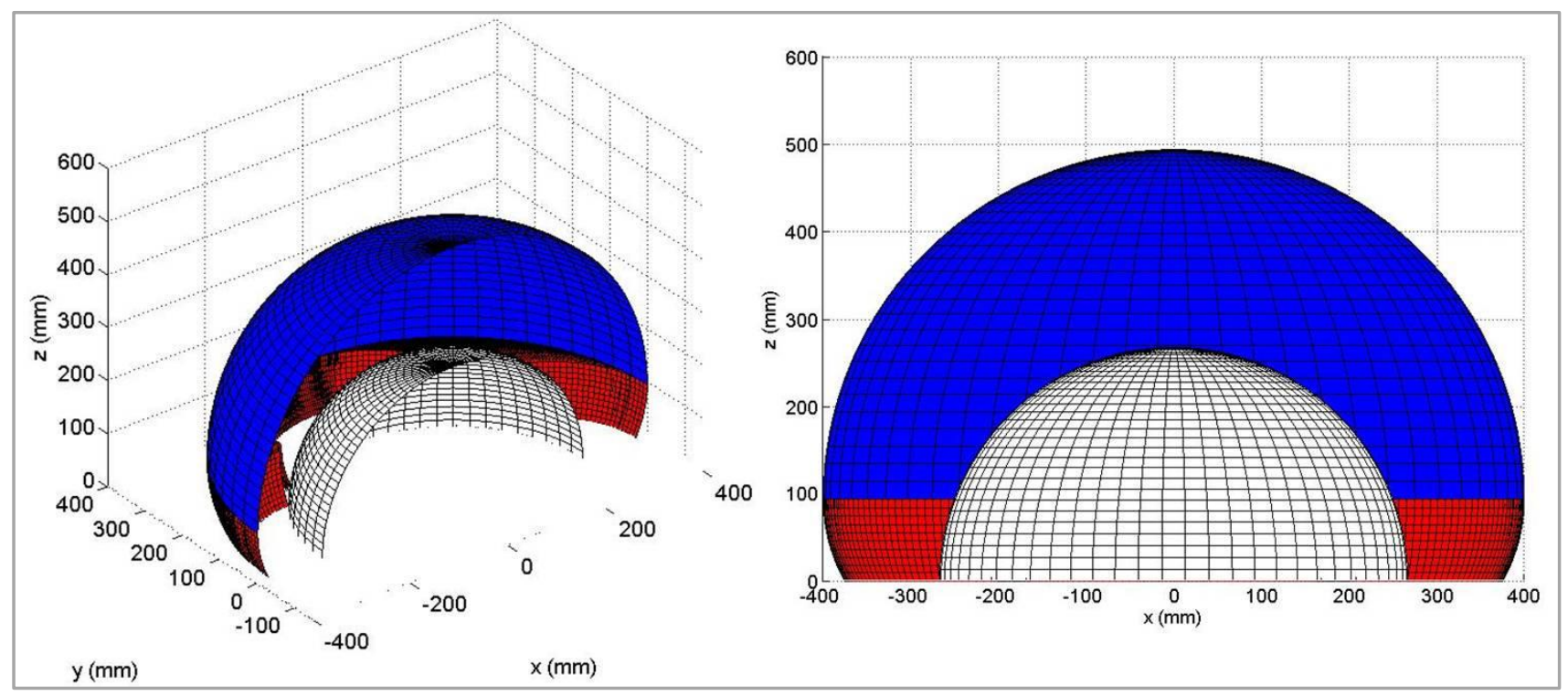

Figure 6: 3D plot of half of the workspace

\section{DESIGN IMPLEMENTATION}

Figure 7 illustrates the flow of signals and energy throughout the robotic arm. A mobile phone camera was used as a visual sensor and was mounted a meter above the arm. After calibration, it was used to obtain the objects coordinates. This is done through the IP Camera application that is installed on the phone, which connects to the computer through $\mathrm{Wi}-\mathrm{Fi}$ and sends a snapshot of the workspace in a $640 \times 480$ resolution. The Matlab code then detects all red, blue and green objects in the snapshot received and calculates their coordinates in pixels. Then using the calibrated equations, pixels are converted into $x$ and $y$ position coordinates. All of the objects are assumed to have the same $z$ coordinate, since they are located in the same horizontal plane (i.e. on the surface of the workbench).

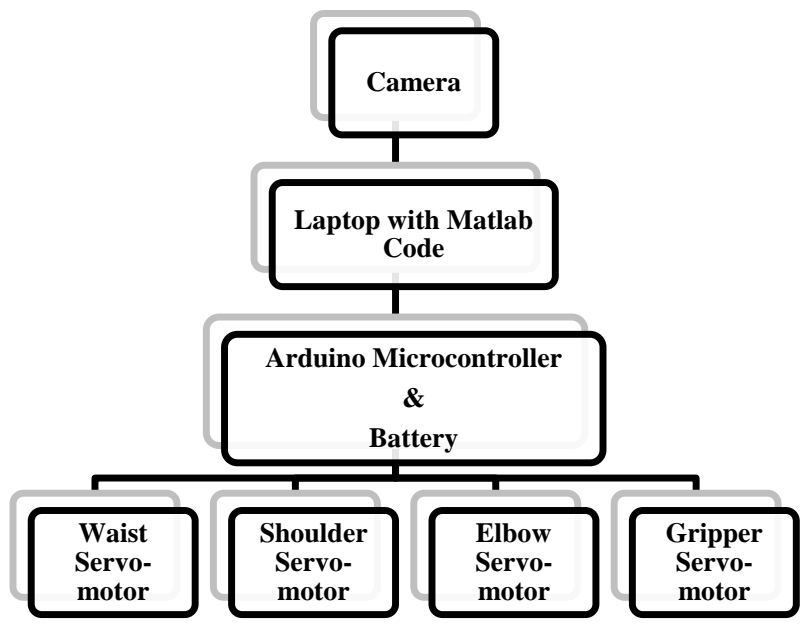

Figure 7: Signal and power flow in the robotic arm

The inverse kinematics equations were integrated into the Matlab code, where the $x$ and $y$ coordinates obtained from the camera are used as an input. The user pre-defines the placing position in the code, which similarly will be substituted into the inverse kinematics equations to generate the required angles for the placing of the object.

Based on the design process described in this paper, a 3 DOF robotic arm was manufactured and is shown in Figure 8 . The next step was the performance of accuracy and repeatability tests on the arm to validate the design.

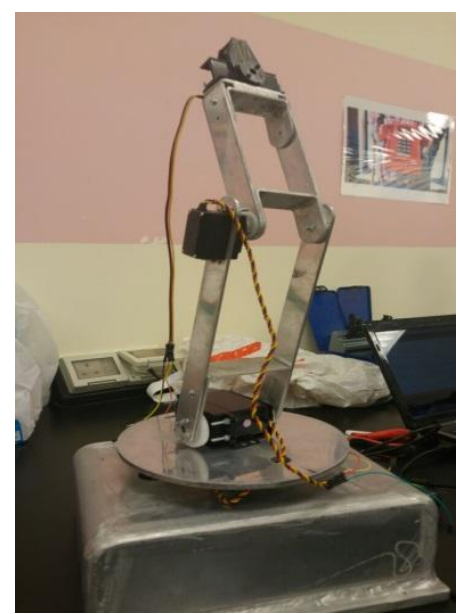

Figure 8: The manufactured 3 DOF robotic arm

To check its repeatability, the arm was commanded to go to the same position at least five times while checking for any variations. To get better results, the experiment was repeated for three different locations. It was noted that the end effector would return to the exact coordinate every time, giving it a repeatability of within $\pm 1 \mathrm{~mm}$.

The accuracy tests were conducted by placing the arm on a grid pattern of known dimensions. First, the image recognition accuracy was tested by comparing the coordinates output by the camera to the actual coordinates of the object, which presented an error of $\pm 1 \mathrm{~mm}$. Similarly, the accuracy of the end effector was tested by finding the difference between the object coordinates and the location of the end effector in its pick/place position, and it was found to be within $\pm 10 \mathrm{~mm}$.

\section{CONCLUCIONS}

The purpose of the study was to design a three degrees of freedom robotic arm that is capable of picking and placing lightweight objects from one to another specific location. The optimal cross-section for the links was found to be a set of two rectangular areas as it has the highest structural strength to weight ratio when compared to solid and hollow circular 
beams. Additionally, the forward and inverse kinematic analyses were carried out and a mathematical equation was created to represent and plot the arm's workspace. Lastly, repeatability and accuracy tests were conducted and the error values for the camera and end effector were found.

For future work, the team will focus on recalibrating the robotic arm to improve its positioning accuracy and setting up a wireless connection for the control system.

\section{REFERENCES}

[1] Shah, J., Rattan, S. S., and Nakra, B.C. 2013. EndEffector Position Analysis Using Forward Kinematics for 5 DOF Pravak Robot Arm. International Journal of Robotics and Automation 2(3) (Sept. 2013), 112 - 116.
[2] Denavit, J. and Hartenberg, R.S. 1955. A Kinematic Notation for Lower-Pair Mechanisms Based on Matrices. Journal of Applied Mechanics 22 (June 1955), 215 - 221.

[3] Fernini B. 2012. Kinematic Modeling and Simulation of a 2-R Robot by Using Solid Works and Verification by MATLAB/Simulink. International Journal of Robotics and Automation 1(2) (June 2012), 78 - 93.

[4] Subhashini, P.V.S., Raju, N.V.S., and Rao, G. V. 2014. Modeling, simulation and analysis of a SCARA robot for deburring of circular components. ARPN Journal of Engineering and Applied Sciences. 9(4) (April 2014), $398-404$.

[5] Craig J. 2016. Introduction to Robotics: Mechanics and Control, 3rd ed 2016. Pearson Education International. 